\title{
Metformin increases endogenous glucose production in non-diabetic individuals and individuals with recent-onset type 2 diabetes
}

\author{
Lars C. Gormsen ${ }^{1}$ (D) $\cdot$ Esben Søndergaard ${ }^{2,3} \cdot$ Nana L. Christensen ${ }^{1} \cdot$ Kim Brøsen $^{4} \cdot$ Niels Jessen $^{5,6,7} \cdot$ Søren Nielsen $^{2,7}$
}

Received: 10 December 2018 / Accepted: 8 March 2019/Published online: 11 April 2019

(C) Springer-Verlag GmbH Germany, part of Springer Nature 2019

\begin{abstract}
Aims/hypothesis Metformin is the endorsed first-line glucose-lowering drug for treating patients with type 2 diabetes but despite more than 50 years of use, no consensus has been reached on its mechanisms of action. In this study, we investigated the glucoselowering effects of metformin in individuals with type 2 diabetes and non-diabetic individuals.

Methods We performed a randomised, placebo-controlled trial in 24 individuals with recent-onset type 2 diabetes (diabetes duration 50 [48] months) who had good glycaemic control $\left(\mathrm{HbA}_{1 \mathrm{c}} 48 \mathrm{mmol} / \mathrm{mmol}\right.$ [6.5\%]). The studies were conducted at Aarhus University Hospital between 2013 and 2016. Participants were randomised to receive either metformin (2000 mg/day, $n=12$, MET group) or placebo ( $n=12$, PLA group) for 90 days, using block randomisation set up by an unblinded pharmacist. Two participants withdrew from the study prior to completion and were replaced with two new participants receiving the same treatment. In addition, we recruited a group of non-diabetic individuals with similar age and BMI ( $n=12$, CONT group), who were all treated with $2000 \mathrm{mg}$ metformin daily. Before and after treatment all individuals underwent studies of whole-body glucose metabolism by non-steady-state $\left[3-{ }^{3} \mathrm{H}\right]$ glucose kinetics, hyperinsulinaemic-euglycaemic clamping, indirect calorimetry, metabolomics, dual x-ray absorptiometry and muscle biopsies. The primary study endpoint was the effect of metformin treatment on lipid kinetics as well as glucose rate of disappearance $\left(R_{\mathrm{d}}\right)$ and endogenous glucose production (EGP).

Results One participant from the CONT group withdrew due to intolerable gastrointestinal side-effects and was excluded from analysis. As expected, metformin treatment lowered fasting plasma glucose (FPG) in the MET group $(\sim 1.5 \mathrm{mmol} / 1, p<0.01)$, whereas no effect was observed in the PLA and CONT groups. Body weight and composition did not change in any of the groups. In both of the metformin-treated groups (MET and CONT), basal glucose $R_{\mathrm{d}}$, EGP and glucagon levels increased by $\sim 30 \%(p<0.05)$ whereas this was not the case in the PLA group.

Conclusions/interpretation Ninety days of metformin treatment resulted in similar increases in EGP and glucose $R_{\mathrm{d}}$ in individuals with recent-onset type 2 diabetes and in non-diabetic control individuals. These results challenge the existing paradigm that metformin primarily acts in the liver by inhibiting EGP, at least in individuals with type 2 diabetes of short duration and who have discretely affected glycaemic status. Whether metformin increases basal glucose $R_{\mathrm{d}}$ by facilitating glucose uptake in other tissues such as the intestines remains to be further clarified.
\end{abstract}

Trial registration ClinicalTrials.gov NCT01729156

Søren Nielsen and Niels Jessen contributed equally to this work.

Electronic supplementary material The online version of this article (https://doi.org/10.1007/s00125-019-4872-7) contains peer-reviewed but unedited supplementary material, which is available to authorised users.

Niels Jessen

Niels.Jessen@biomed.au.dk

1 Department of Nuclear Medicine \& PET Centre, Aarhus University Hospital, Aarhus, Denmark

2 Department of Endocrinology, Aarhus University Hospital, Aarhus, Denmark

3 Danish Diabetes Academy, Odense University Hospital, Odense, Denmark
4 Department of Pharmacology, Odense University Hospital, Odense, Denmark

5 Department of Clinical Pharmacology, Aarhus University Hospital, Aarhus, Denmark

6 Department of Biomedicine, Aarhus University, Aarhus, Denmark

7 Steno Diabetes Center Aarhus, Aarhus University Hospital, DK 8200 Aarhus, Denmark 


\section{Research in context}

\section{What is already known about this subject?}

- Metformin lowers fasting plasma glucose and $\mathrm{HbA}_{1 \mathrm{c}}$ in individuals with type 2 diabetes

- Previous studies in individuals with poorly controlled diabetes have demonstrated that metformin reduces hepatic, and therefore endogenous, glucose production

- However, studies in insulin-sensitive individuals have indicated that metformin may instead increase glucose uptake, triggering a compensatory increase in endogenous glucose production

What is the key question?

- What is the effect of metformin on endogenous glucose production in individuals with recent-onset and wellcontrolled type 2 diabetes and how does it compare with non-diabetic individuals?

What are the new findings?

- Metformin increased basal glucose disposal, glucagon levels and endogenous glucose production both in individuals with type 2 diabetes and non-diabetic individuals

How might this impact on clinical practice in the foreseeable future?

- The glucose-lowering effect of metformin does not involve a reduction of endogenous glucose production in newly diagnosed type 2 diabetes

Funding This study was supported by grants from The Danish Council for Independent Research | Medical Sciences, Aase Danielsen Fund, the Novo Nordisk Foundation, the Danish Diabetes Association and the Danish Diabetes Academy supported by the Novo Nordisk Foundation.

Keywords Clinical trial · Glucose kinetics · Metformin · Type 2 diabetes

$\begin{array}{ll}\begin{array}{l}\text { Abbreviations } \\ \text { CONT }\end{array} & \begin{array}{l}\text { Non-diabetic individuals given metformin } 1000 \mathrm{mg} \\ \text { twice daily }\end{array} \\ \text { EGP } & \text { Endogenous glucose production } \\ \text { FPG } & \text { Fasting plasma glucose } \\ \text { MET } & \text { Participants with type } 2 \text { diabetes given metformin } \\ & 1000 \text { mg twice daily } \\ \text { NOGD } & \text { Non-oxidative glucose disposal } \\ \text { OCT1 } & \text { Organic cation transporter-1 } \\ \text { PLA } & \text { Participants with type } 2 \text { diabetes given placebo } \\ R_{\mathrm{d}} & \text { Rate of disappearance }\end{array}$

\section{Introduction}

Metformin is currently the preferred and endorsed first-line glucose-lowering drug prescribed for patients with type $2 \mathrm{di}$ abetes [1]. It is generally well-tolerated, has few serious adverse effects and costs a fraction of recently introduced glucose-lowering agents. Although numerous large-scale studies have documented consistent decreases in $\mathrm{HbA}_{1 \mathrm{c}}$ and fasting plasma glucose (FPG) levels after treatment initiation, no consensus has been reached on its mechanisms of action.
A commonly proposed mechanism of action for the glucose-lowering effect of metformin is through reduced hepatic or endogenous glucose production (EGP) [2]. Studies have shown considerable reductions in EGP (15-25\%) after metformin treatment in individuals with poor glucose control [2-4] but this has not been a consistent finding [5]. In fact, when exclusively double-blinded, placebo-controlled studies are analysed, metformin only lowers EGP marginally $(\sim 5 \%)$ and not to an extent that can explain the concomitant decrease in FPG [6], and may even increase EGP [7].

Therefore, the aim of this study was to compare the effects of 3 months of metformin treatment on lipid (previously published [8]) and glucose metabolism between patients with recent-onset type 2 diabetes and non-diabetic individuals. It was our main hypothesis that metformin increases basal non-oxidative glucose disposal (NOGD) and, secondary to this, EGP, regardless of blood glucose status. Our main endpoint was the assessment of whole-body glucose metabolism by $\left[3-{ }^{3} \mathrm{H}\right]$ glucose kinetics.

\section{Methods}

The study was performed in accordance with the Declaration of Helsinki after approval by the Central Denmark Committee 
on Health Research (no. 1-10-72-522-12) and the Danish Medicines Agency (EudraCT2012-000808-16). The local GCP-unit at Aarhus University Hospital monitored the study. All participants signed a written informed consent prior to inclusion.

Study design In short, 24 participants with recent-onset type 2 diabetes were randomised to receive either metformin (1000 mg twice daily; MET group) or placebo (PLA group) for 3 months while 12 non-diabetic individuals were treated with metformin (1000 mg twice daily; CONT group). All participants underwent identical studies of whole-body glucose metabolism (steady-state $\left[3-{ }^{3} \mathrm{H}\right]$ glucose and indirect calorimetry) as well as non-targeted metabolomics analysis of plasma samples and skeletal muscle mitochondrial protein expression before and after $90 \pm 3$ days treatment (electronic supplementary material [ESM] Fig. 1). Participant recruitment, demography and methods are described in detail in the ESM Methods and our previous paper reporting lipidrelated metabolism [8].

Statistics Statistical analyses were performed using a hierarchical mixed linear model with compound symmetry covariance structure, including one within-factor (time: overall mean change between study days), one between-factor (group: PLA, MET and CONT) and an interaction term (group $\times$ time: indicating whether mean change during the study was different between groups). For the primary glucose metabolic variables, additional mixed model analyses for two groups were performed comparing effects of metformin treatment in individuals with type 2 diabetes (PLA vs MET) and effects of diabetes status in all participants treated with identical doses of metformin (MET vs CONT). Unless otherwise stated, values are given as mean $\pm \mathrm{SD}$. A $p$ value $<0.05$ was considered significant. All analyses were performed in SPSS version 21 (IBM, Armonk, NY, USA).

\section{Results}

FPG is decreased by metformin treatment FPG $(p<0.001)$ and insulin levels $(p=0.06)$ were lower in the CONT group than in the PLA and MET groups (ESM Fig. 2). As expected, metformin treatment reduced FPG significantly $(\sim 1.5 \mathrm{mmol} / \mathrm{l}$; $p<0.01)$ in the MET group whereas no changes were observed in the PLA and CONT groups (Table 1).

Basal EGP, NOGD and rate of glucose disappearance is increased by metformin treatment We first explored the effect of metformin on EGP in the basal state (Fig. 1a) and found a significant interaction revealing a stimulatory rather than inhibitory effect in both MET and CONT groups (mixed model time vs group interaction $p=0.02$ ) (see Table 1 for values). An additional post hoc analysis comparing MET and CONT groups convincingly demonstrated a similar stimulatory effect of metformin on EGP regardless of blood glucose status (mixed model time $p=0.02$ ). In line with this, the basal rate of disappearance $\left(R_{\mathrm{d}}\right)$ for glucose (Fig. 1c) was unaffected in the PLA group and increased to a similar extent in the metformin-treated groups (MET and CONT).

Second, we explored the effect of hyperinsulinaemia and found a smaller degree of group-related differences (Fig. 1b,d and Table 1). As expected, EGP during hyperinsulinaemia was lower overall in non-diabetic control individuals than in individuals with type 2 diabetes and was suppressed to a greater extent than in individuals with type 2 diabetes (Table 1). Glucose $R_{\mathrm{d}}$ during hyperinsulinaemia also increased in both MET and CONT groups and was unaffected in the PLA group.

Basal resting energy expenditure and glucose and lipid oxidation rates were comparable among groups and were unaffected by treatment (see ESM Table 1). Basal NOGD was significantly increased in both metformin-treated groups (Table 1).

Glucagon is stimulated by metformin treatment and cortisol is unaffected To test whether the increase in EGP was caused by a counter-regulatory hormonal response, we measured glucagon and cortisol under basal conditions. Glucagon levels were elevated in the PLA group compared with the MET and CONT groups (Table 1). In a post hoc analysis comparing PLA and MET groups, no effect of treatment was observed. However, when comparing MET and CONT groups, metformin treatment induced a $\sim 30 \%$ increase in glucagon levels in both groups as reflected by a mixed model time $p=0.01$.

Cortisol levels were similar among groups and remained unaffected by treatment (Table 1), whereas metabolomics showed diabetes-related differences, particularly in relation to lipid metabolism (ESM Table 2). Two skeletal mitochondrial proteins were also decreased by metformin treatment (ESM Fig. 3).

\section{Discussion}

This placebo-controlled, double-blinded, parallel study demonstrates that 3 months of treatment with metformin lowers fasting plasma glucose levels in individuals with recent-onset type 2 diabetes but not in non-diabetic individuals with similar age and BMI. However, both non-diabetic individuals and individuals with type 2 diabetes showed similar concurrent increases in basal NOGD, EGP and glucagon levels when treated with metformin, challenging the existing paradigm of metformin action on hepatic glucose production.

Our data thus furthers the notion that metformin action in type 2 diabetes is not confined to the liver. We believe this observation may have several explanations. First, it is 
Table 1 Characteristics of participants

\begin{tabular}{|c|c|c|c|c|c|c|c|c|c|}
\hline \multirow[t]{2}{*}{ Characteristic } & \multicolumn{2}{|l|}{ PLA } & \multicolumn{2}{|l|}{ MET } & \multicolumn{2}{|l|}{ CONT } & \multicolumn{3}{|l|}{$p$ value } \\
\hline & Study day 1 & Study day 2 & Study day 1 & Study day 2 & Study day 1 & Study day 2 & Group & Time & $\begin{array}{l}\text { Group } \times \\
\text { time }\end{array}$ \\
\hline \multicolumn{10}{|l|}{ Baseline characteristics } \\
\hline Sex ( $n$ male $/ n$ female) & $9 / 3$ & $9 / 3$ & $6 / 6$ & $6 / 6$ & $6 / 6$ & $6 / 5$ & 0.38 & & \\
\hline Age, years & $60(5)$ & & $64(5)$ & & $62(6)$ & & 0.22 & & \\
\hline BMI, $\mathrm{kg} / \mathrm{m}^{2}$ & $31.2(4.3)$ & & $30.3(5.7)$ & & $27.3(4.1)$ & & 0.12 & & \\
\hline Waist/hip circumference & $1.01(0.11)$ & & $0.97(0.10)$ & & $0.90(0.13)$ & & 0.10 & & \\
\hline Diabetes duration, months & $58(54)$ & & $43(43)$ & & & & 0.46 & & \\
\hline Previous metformin treatment, $n /$ total & $9 / 12$ & & $7 / 12$ & & & & 0.67 & & \\
\hline Concomitant statin treatment, $n /$ total & $8 / 12$ & & $7 / 12$ & & $2 / 12 *$ & & 0.03 & & \\
\hline $\begin{array}{l}\text { Other oral glucose-lowering } \\
\text { treatment, } n / \text { total }\end{array}$ & $3 / 12$ & & $1 / 12$ & & & & 0.59 & & \\
\hline $\mathrm{HbA}_{1 \mathrm{c}}, \mathrm{mmol} / \mathrm{mmol}$ & $45(6)$ & $\mathrm{ND}$ & $51(6)^{\dagger}$ & ND & $37(3)^{*}$ & ND & $<0.001$ & & \\
\hline $\mathrm{HbA}_{1 \mathrm{c}}, \%$ & $6.2(0.5)$ & $\mathrm{ND}$ & $6.8(0.5)^{\dagger}$ & & $5.6(0.3)^{*}$ & & $<0.001$ & & \\
\hline Total cholesterol, mmol/1 & $4.2(0.9)$ & & $4.5(1.4)$ & & $5.7(1.1)^{*}$ & & 0.01 & & \\
\hline HDL-cholesterol, mmol/1 & $1.2(0.4)$ & & $1.4(0.5)$ & & $1.8(0.5)^{*}$ & & 0.01 & & \\
\hline LDL-cholesterol, mmol/1 & $2.1(0.9)$ & & $2.4(1.2)$ & & $3.4(0.9)^{*}$ & & 0.01 & & \\
\hline Weight, kg & $93.8(17.4)$ & $93.3(16.9)$ & $88.6(16.4)$ & $88.2(16.3)$ & $79.3(14.9)$ & $79.6(15.3)$ & 0.10 & 0.30 & 0.97 \\
\hline Lean body mass, $\mathrm{kg}$ & $60.6(12.2)$ & $60.3(12.1)$ & $53.9(8.0)$ & $53.7(8.2)$ & $51.0(9.9)$ & $51.0(9.7)$ & 0.07 & 0.18 & 0.73 \\
\hline \multicolumn{10}{|l|}{ Metabolism and hormones } \\
\hline FPG, mmol/l & $6.9(1.0)$ & $7.0(2.0)$ & $7.9(1.8)$ & $6.4(1.1)^{\dagger}$ & $5.4(0.3)^{*}$ & $5.2(0.4)$ & 0.001 & 0.01 & 0.003 \\
\hline Basal insulin, pmol/1 & $83(32)$ & $81(46)$ & $68(49)$ & $60(38)$ & $44(23)$ & $44(33)$ & 0.06 & 0.30 & 0.55 \\
\hline Basal glucagon, pmol/1 & $10.2(5.2)$ & $9.3(4.5)$ & $5.1(1.7)$ & $6.9(3.6)$ & $4.9(3.4)$ & $6.2(3.8)$ & 0.01 & 0.26 & 0.21 \\
\hline Basal cortisol, ng/ml & $113(46)$ & $108(33)$ & $122(44)$ & $103(33)$ & $130(60)$ & $141(69)$ & 0.29 & 0.63 & 0.40 \\
\hline$M$ value, $\mathrm{mg} \mathrm{kg}^{-1} \min ^{-1}$ & $1.30(0.62)$ & $1.54(1.07)$ & $1.26(1.05)$ & $1.57(0.64)$ & $2.18(0.69)^{*}$ & $2.15(0.66)$ & 0.03 & 0.15 & 0.45 \\
\hline Basal EGP, $\mathrm{mg} \mathrm{kg}^{-1} \mathrm{~min}^{-1}$ & $1.19(0.36)$ & $1.12(0.45)$ & $1.23(0.52)$ & $1.70(0.46)$ & $1.43(0.57)$ & $1.72(0.31)$ & 0.03 & 0.02 & 0.07 \\
\hline Clamp EGP, $\mathrm{mg} \mathrm{kg}^{-1} \mathrm{~min}^{-1}$ & $0.81(0.39)$ & $0.55(0.36)$ & $0.86(0.47)$ & $1.01(0.44)$ & $0.50(0.39)$ & $0.62(0.35)$ & 0.02 & 0.98 & 0.13 \\
\hline EGP suppression during clamp, $\%$ & $32(21)$ & $48(31)$ & $25(40)$ & $37(25)$ & $62(26)^{*}$ & $64(18)^{*}$ & 0.01 & 0.09 & 0.54 \\
\hline Basal glucose $R_{\mathrm{d}}, \mathrm{mg} \mathrm{kg}^{-1} \min ^{-1}$ & $1.36(0.28)$ & $1.30(0.32)$ & $1.32(0.42)$ & $1.78(0.43)$ & $1.50(0.63)$ & $1.74(0.30)$ & 0.11 & 0.02 & 0.07 \\
\hline Clamp glucose $R_{\mathrm{d}}, \mathrm{mg} \mathrm{kg}^{-1} \mathrm{~min}^{-1}$ & $2.21(0.64)$ & $2.43(1.00)$ & $2.21(0.87)$ & $2.58(0.65)$ & $2.91(0.99)$ & $3.01(0.77)$ & 0.10 & 0.06 & 0.59 \\
\hline Basal NOGD, $\mathrm{mg} \mathrm{kg}^{-1} \min ^{-1}$ & $0.42(0.37)$ & $0.22(0.53)$ & $0.20(0.77)$ & $0.92(0.68)^{\dagger}$ & $0.40(0.87)$ & $0.89(0.81)^{\dagger}$ & 0.37 & 0.02 & 0.02 \\
\hline
\end{tabular}

All values are mean (SD) except for ratios

All initial statistical analyses were done using a mixed model except for baseline comparisons of dichotomous variables, which were analysed by a $\chi^{2}$ test. Group $p$ value indicates whether there is a difference between all groups; time $p$ value indicates the mean change between study day 1 and study day 2 values; and group $\times$ time indicates whether the mean changes are different between groups

Post hoc comparisons of differences between groups were done by unpaired $t$ tests: $* p<0.05$ vs PLA and MET; ${ }^{\dagger} p<0.05$ vs PLA

important to stress that our group of participants with recentonset type 2 diabetes had significantly better glycaemic control ( $\mathrm{HbA}_{1 \mathrm{c}}$ levels $\sim 42-53 \mathrm{mmol} / \mathrm{mmol}$ [6-7\%]) than those enrolled in most of the previously published studies of metformin action $\left(\mathrm{HbA}_{1 \mathrm{c}}\right.$ levels $\sim 86 \mathrm{mmol} / \mathrm{mmol}$ [10\%]) [6]. It is possible that a longer duration of diabetes gradually results in alterations in hepatic glucose production, which we were unable to detect in our relatively mildly affected participant cohort. Second, individuals with mutations in SLC22A1, which encodes organic cation transporter-1 (OCT1), who have reduced capacity to transport the drug into the hepatocytes, still benefit from treatment as judged by decreased $\mathrm{HbA}_{1 \mathrm{c}}$ levels [9].
Similarly, the increased EGP in response to metformin treatment in non-diabetic individuals reported by Christensen et al was independent of OCT1 function [7]. The main effect of metformin on FPG would, therefore, appear to be an extrahepatic one and the increase in EGP to be a result of a counter-regulatory response. This notion is supported by the avid glucose and $\left[{ }^{18} \mathrm{~F}\right]$ fluorodeoxyglucose uptake observed in the bowel of metformin-treated individuals undergoing oncological PET/CT, and could explain the observed increase in NOGD in the basal non-insulin-stimulated state. Metformin is transported into enterocytes via organic cation transporters, plasma membrane monoamine transporter (PMAT) and OCT1 

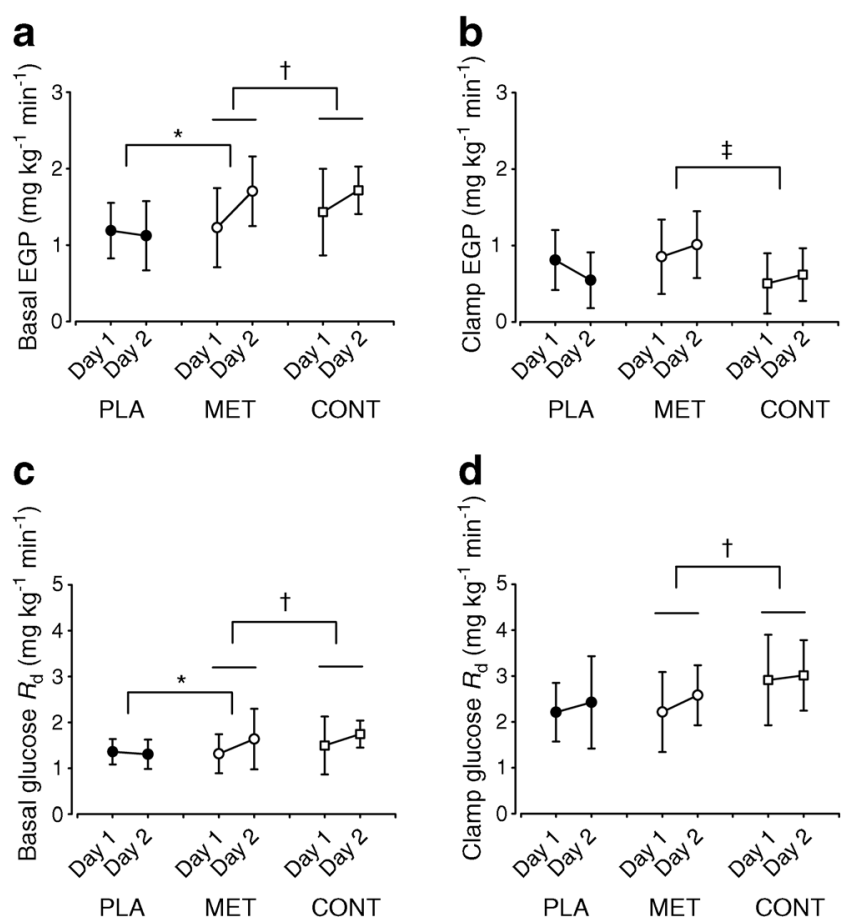

Fig. 1 Glucose metabolism assessed by steady-state $\left[3-{ }^{3} \mathrm{H}\right]$ glucose kinetics on study days 1 and 2 . The $p$ values refer to significant $(p<0.05)$ post hoc analyses: $* p<0.05$ for PLA vs MET (mixed model term of interest, group vs time interaction); ${ }^{\dagger} p<0.05$ for MET vs CONT (mixed model term of interest, time). ${ }^{*} p<0.05$ for MET vs CONT (mixed model term of interest, group) (a) Glucose rate of appearance (and therefore, by definition, EGP). As seen, metformin treatment resulted in increased EGP in participants with type 2 diabetes (PLA vs MET, group vs time interaction $p=0.02$ ), When comparing participants treated with metformin, both with (MET) and without diabetes (CONT), EGP increased in a similar fashion (mixed model, time $p<0.05$ ). (b) EGP during the hyperinsulinaemiceuglycaemic clamp, which, as anticipated, was lower in metformin-treated non-diabetic individuals than in individuals with type 2 diabetes (MET vs CONT, group $p=0.01$ ). (c) Basal glucose $R_{\mathrm{d}}$ was affected in a similar way to EGP, with increased levels seen in metformin-treated participants with type 2 diabetes vs placebo-treated participants (PLA vs MET, group vs time interaction $p=0.01$ ) and a comparable increase in both diabetic (MET) and non-diabetic (CONT) individuals treated with metformin (mixed model, time $p=0.01$ ). (d) Clamp glucose $R_{\mathrm{d}}$ showed only minor differences between groups, although glucose $R_{\mathrm{d}}$ increased in both diabetic (MET) and non-diabetic (CONT) individuals treated with metformin (mixed model, time $p=0.04$ ). Data are mean $\pm \mathrm{SD}$

[10], and is subsequently transported via the portal circulation to the liver in concentrations approximately fourfold those found systemically. Consequently, liver concentrations of metformin are significantly greater than peripheral tissue concentrations and this has been a major argument in favour of the hypothesis that the liver is the main target organ of metformin. However, by this logic, the enterocytes are in all likelihood exposed to even greater concentrations of metformin and any effect of metformin on enterocyte function should therefore translate into significant whole-body metabolic alterations. Indeed, it has been estimated that enterocyte metformin concentrations in the distal ileum may be $\sim 300$-fold greater than systemic metformin concentrations [11].
The decrease in FPG during metformin treatment is likely to be the cause of the observed increase in glucagon levels. Lower circulating glucose levels stimulate pancreatic glucagon secretion, which is roughly linearly and inversely correlated to glucose concentrations within the range of $0-10 \mathrm{mmol} / \mathrm{l}$ [12]. Importantly, the increased glucagon level may explain the increase in EGP during metformin treatment. There appears to be a linear doseresponse relationship between sinusoidal glucagon levels and hepatic glucose production, at least in the low basal range (reviewed in [13]). Since glucagon concentrations in our experiments increased $18-30 \%$ during metformin treatment, a roughly similar increase in EGP could be expected and was indeed observed.

Although we did use gold standard methods to assess glucose homeostasis, our study is limited by the lack of direct evidence that the decrease in circulating glucose and increased basal NOGD is caused by increased intestinal uptake. The duration of the trial is also a limitation, as participants were only treated for 3 months. The short treatment duration led to the expected changes in FPG but is most likely too short to detect changes in $\mathrm{HbA}_{1 \mathrm{c}}$, as $\mathrm{HbA}_{1 \mathrm{c}}$ is generally not considered sensitive to changes in plasma glucose in clinical trials lasting $<12$ weeks.

In summary, data from the present study demonstrate that treatment with metformin increases basal glucose $R_{\mathrm{d}}$, NOGD and EGP levels in a similar fashion in non-diabetic individuals and individuals with recent-onset and well-controlled type 2 diabetes. These observations challenge the notion that metformin primarily acts in the liver, at least in the early stages of type 2 diabetes. It is therefore possible that the initial glucoselowering effect of metformin may be to facilitate intestinal glucose storage.

Acknowledgements The authors would like to thank L. Kvist and S. Sørensen (Medical Research Laboratories, Aarhus University Hospital) for excellent technical help during the studies.

Data availability The datasets generated during and/or analysed during the current study are available from the corresponding author on reasonable request.

Funding The study was supported by grants from The Danish Council for Independent Research | Medical Sciences, Aase Danielsen Fund, the Novo Nordisk Foundation, the Danish Diabetes Association and the Danish Diabetes Academy supported by the Novo Nordisk Foundation.

Duality of interest The authors declare that there is no duality of interest associated with this manuscript.

Contribution statement All authors conceptualised the study, wrote the protocol, researched the data and contributed to discussion. LCG wrote the manuscript and ES, NLC, KB, NJ and SN reviewed and edited the manuscript. LCG, NJ and SN are the guarantors of this work and, as such, had full access to all the data in the study and take responsibility for the integrity of the data and the accuracy of the data analysis. All authors approved the final version of the manuscript. 


\section{References}

1. Inzucchi SE, Bergenstal RM, Buse JB et al (2012) Management of hyperglycemia in type 2 diabetes: a patient-centered approach: position statement of the American Diabetes Association (ADA) and the European Association for the Study of Diabetes (EASD). Diabetes Care 35(6):1364-1379. https://doi.org/10.2337/dc12-0413

2. Hundal RS, Krssak M, Dufour S et al (2000) Mechanism by which metformin reduces glucose production in type 2 diabetes. Diabetes 49(12):2063-2069. https://doi.org/10.2337/diabetes.49.12.2063

3. Inzucchi SE, Maggs DG, Spollett GR et al (1998) Efficacy and metabolic effects of metformin and troglitazone in type II diabetes mellitus. N Engl J Med 338(13):867-872. https://doi.org/10.1056/ NEJM199803263381303

4. Perriello G, Misericordia P, Volpi E et al (1994) Acute antihyperglycemic mechanisms of metformin in NIDDM. Evidence for suppression of lipid oxidation and hepatic glucose production. Diabetes 43(7):920-928. https://doi.org/10.2337/diab. 43.7.920

5. Hother-Nielsen O, Schmitz O, Andersen PH, Beck-Nielsen H, Pedersen O (1989) Metformin improves peripheral but not hepatic insulin action in obese patients with type II diabetes. Acta Endocrinol 120(3):257-265. https://doi.org/10.1530/acta.0.1200257

6. Natali A, Ferrannini E (2006) Effects of metformin and thiazolidinediones on suppression of hepatic glucose production and stimulation of glucose uptake in type 2 diabetes: a systematic review. Diabetologia 49(3):434-441. https://doi.org/10.1007/ s00125-006-0141-7

7. Christensen MM, Hojlund K, Hother-Nielsen O et al (2015) Endogenous glucose production increases in response to metformin treatment in the glycogen-depleted state in humans: a randomised trial. Diabetologia 58(11):2494-2502. https://doi.org/10.1007/ s00125-015-3733-2

8. Gormsen LC, Søndergaard E, Christensen NL et al (2018) Metformin does not affect postabsorptive hepatic free fatty acid uptake, oxidation or resecretion in humans: a 3-month placebocontrolled clinical trial in patients with type 2 diabetes and healthy controls. Diabetes Obes Metab 20(6):1435-1444. https://doi.org/ 10.1111/dom.13244

9. Zhou K, Donnelly LA, Kimber CH et al (2009) Reduced-function SLC22A1 polymorphisms encoding organic cation transporter 1 and glycemic response to metformin: a GoDARTS study. Diabetes 58(6):1434-1439. https://doi.org/10.2337/db08-0896

10. Rena G, Hardie DG, Pearson ER (2017) The mechanisms of action of metformin. Diabetologia 60(9):1577-1585. https://doi.org/10. 1007/s00125-017-4342-z

11. Bailey CJ, Wilcock C, Scarpello JH (2008) Metformin and the intestine. Diabetologia 51(8):1552-1553. https://doi.org/10.1007/ s00125-008-1053-5

12. Gerich JE, Charles MA, Grodsky GM (1974) Characterization of the effects of arginine and glucose on glucagon and insulin release from the perfused rat pancreas. J Clin Invest 54(4):833-841. https:// doi.org/10.1172/JCI107823

13. Cherrington AD (1999) Banting Lecture 1997. Control of glucose uptake and release by the liver in vivo. Diabetes 48(5):1198-1214. https://doi.org/10.2337/diabetes.48.5.1198

Publisher's note Springer Nature remains neutral with regard to jurisdictional claims in published maps and institutional affiliations. 